\title{
High Energy Density Soft X-ray Momentum Coupling to Comet Analogs for NEO Mitigation
}

\author{
J. L. Remo ${ }^{1,2,3}$, R. J. Lawrence ${ }^{4}$, S. B. Jacobsen ${ }^{2}$, \\ and M. D. Furnish ${ }^{4}$
}

${ }^{1}$ Departments of Astronomy and ${ }^{2}$ Earth and Planetary Sciences, Harvard University, 20 Oxford St. and ${ }^{3}$ Harvard-Smithsonian Center for Astrophysics, 60 Garden St., Cambridge MA, 02138, USA (jremo@cfa.harvard.edu) and ${ }^{4}$ Sandia National Laboratories, P.O. Box 5800, Albuquerque NM, 87185-1185, USA (mdfurni@sandia.gov)

\begin{abstract}
We applied MBBAY high fluence pulsed radiation intensity driven momentum transfer analysis to calculate X-ray momentum coupling coefficients $C_{M}=(P a-s) /\left(J / m^{2}\right)$ for two simplified comet analog materials: i) water ice, and ii) $70 \%$ water ice and $30 \%$ distributed olivine grains. The momentum coupling coefficients $\left(\mathrm{C}_{\mathrm{M}}\right)_{\max }$ of $50 \times 10^{-5} \mathrm{~s} / \mathrm{m}$, are about an order of magnitude greater than experimentally determined and computed MBBAY values for meteoritic materials that are analogs for asteroids. From the values for comet analog materials we infer applied energies (via momentum transfer) required to deflect an Earth crossing comet from impacting Earth by a sufficient amount $(\sim 1 \mathrm{~cm} / \mathrm{s})$ to avert collision $\sim$ a year in advance. Comet model calculations indicate for $C_{M}=5 \times 10^{-4} \mathrm{~s} / \mathrm{m}$ the deflection of a $2 \mathrm{~km}$ comet with a density $600 \mathrm{~kg} / \mathrm{m}^{3}$ by $1 \mathrm{~cm} / \mathrm{s}$ requires an applied energy on the target surface of $5 \times 10^{13} \mathrm{~J}$, the equivalent of $12 \mathrm{kT}$ of TNT. Depending on the geometrical configuration of the interaction the explosive yield required could be an order of magnitude higher.
\end{abstract}

\section{Introduction}

This work analyzes requirements for deflecting potentially hazardous comets with a high probability of Earth impact. It does so by extending previous research $[1,2,3]$ on near-Earth asteroid (NEA) orbital deflection by high intensity pulsed X-rays and neutrons. Computational models simulate high power pulsed nuclear explosive radiation emission, target irradiation effects and subsequent surface blow-off ejection to generate momentum to modify orbits of nearEarth object (NEO) targets. These orbital perturbations can mitigate threats from potentially hazardous NEOs. Other methods of orbital perturbation have been described in the recent literature [4 - 7], but will not be discussed here.

Previous analysis was facilitated by spectral relationships between asteroids and meteorites as asteroid fragments representing different classes of recovered meteorites [8]. The implied relationship became definitive with the sampling of the near-Earth asteroid (NEA) Itokawa by 
the unmanned Hayabusa space mission which recovered samples in 2005 that matched LL chondrites, the most common meteorite type found on Earth. Therefore, laboratory studies of the stable mineral and physical structure of most meteorite types were used to interpret and model the physical properties of asteroids. This facilitated detailed material properties data input into computational modeling of NEAs as well as providing real asteroid target materials for high energy density experiments using the Sandia Z machine, which produces plasma generated Xrays that can be used to simulate nuclear explosive emissions [1].

Here we apply similar analytic and (future) experimental methodologies to simple putative comet models. However, the inherent diversity and/or continual changes in comet composition, density and orbital perturbations from non-gravitational dynamics such as outgassing, combined with extreme difficulties in their remote exploration reconnaissance and recovery doesn't permit reliable chemical and physical descriptions of distinct archetypical classes of comets as in the case of asteroids with meteorites. Nonetheless, to initially quantify the issue of diversity two simplified comet models are considered for calculation; one is composed of pure water ice and the other is $70 \%$ ice and $30 \%$ olivine $\left(\mathrm{Mg}_{2} \mathrm{SiO}_{4}\right)$ uniformly distributed within the comet model. As a reference model a $100 \%$ olivine target model can be extrapolated. Olivine was chosen because it is ubiquitous both in the solar system and in interplanetary space and is observed in comets, meteorites, lunar and Martian rocks and in the discs of young stars.

All models assume zero porosity and uniform, homogeneous and isotropic distribution of target materials with the porosity only affecting overall density. Since the X-ray radiation driven ablation modeled here only significantly interacts with the comet surface to depths of $\sim$ mm or less depending on the X-ray energy, the internal structure and composition, e.g. rocky core, is not critical except for contributing to the overall mass.

\section{Recent discoveries}

Recent discoveries indicate numerous icy bodies in the main asteroid belt in addition to the huge population of Kuiper belt (KBO) and an even more extensive population of Oort cloud comet-like objects. Also, recent reconnaissance of Pluto and its companions by the New Horizons mission suggests high energy radiative transfer work on primarily icy bodies is important for understanding early phases of solar system evolution when an active proto Sun emitted substantial amounts of ultraviolet and soft X-ray radiation. It also is important for comet NEO hazard mitigation where the X-ray driven surface plasma jetting of target materials from near surface $(\sim \mathrm{mm})$ layers provides recoil momentum critical for NEO orbital deflection of an Earth threatening object while minimally affecting the NEO structural integrity [1]. This applies to both comets and asteroids. A range of chemical and physical processes acts on primarily volatile and dusty materials during the formation and evolution of comet nuclei surfaces, especially at perihelia. This suggests they are highly susceptible to high energy density radiation driven surface processing which affects its chemistry and structure. For example, all proto comet material are thought to have originated in the interstellar medium (ISM) but crystalline silicates have not been detected because they are thought to have been formed later by X-ray, UV, and visible radiation emitted from an early stage and active protosun. 


\section{Comet modeling}

Despite an assumed common origin and primarily water ice composition, comets vary widely in composition and structure. There presently isn't an archetypical or evolutionary classification scheme as there is for asteroids (fragments) serving as progenitors of meteorites, except for relatively small and rare Martian and lunar origin meteorites that are abundant on Earth and have been extensively studied and classified [8]. Currently, the single most common characteristic of comets appears to be a predominance, $\sim 60 \%$ or greater, of water ice followed by olivine and carbon dust and small amounts of a great variety of simple and complex hydrocarbons [9 -11]. Diversity among comets may be especially pronounced for closer Kuiper belt objects. The Rosseta interception orbiter and lander mission was found to have a diverse structural landscape primarily of water ice with copious amounts of dust, grains, and complex organic molecules with a bulk density of $\sim 470 \mathrm{~kg} / \mathrm{m}^{3}$ [9]. This low density suggests considerable porosity and also argues against a dense (rocky) core.

In modeling comets, a simple parametric approach isolates the effects of the most relevant parameters. A binary molecular mixture of water ice as the primary component and olivine as the secondary is used. The grains and dust are taken into account by mixing into the ice olivine crystals $\left(\mathrm{Mg}_{2} \mathrm{SiO}_{4}\right.$, or pure forsterite) to comprise $30 \%$ mass fraction of the whole. For reference, irradiation of a $100 \%$ ice target is also computed. This approach provides parametric estimates of ice and ice/olivine mixture in response to high intensity X-ray radiation driven momentum coupling. Later more detailed chemical compositions and distribution of secondary compounds can be incorporated into the numerical models, if necessary.

\section{Momentum coupling equation for nuclear interdiction}

A catastrophic Earth-crossing comet collision with Earth can potentially be avoided by introducing a small velocity change $\Delta \mathrm{v}(\sim \mathrm{cm} / \mathrm{s})$ long beforehand ( $\sim$ year), depending on its mass, position, and velocity. This required velocity change is small relative to the orbital velocity of the comet, which can be many tens of kilometers per second. If a nuclear device is used to irradiate the comet surface with energy $\mathrm{E}$, this small velocity change $\Delta \mathrm{v}$ may be expressed as:

$$
\Delta \mathrm{v}=\mathrm{C}_{\mathrm{M}} \mathrm{E} / \mathrm{M}
$$

where $\mathrm{C}_{\mathrm{M}}$ is the momentum coupling coefficient defined by

$$
\mathrm{C}_{\mathrm{M}} \equiv(\text { force-time } / \text { area }) / \mathrm{F}=(\mathrm{Pa}-\mathrm{s}) /\left(\mathrm{J} / \mathrm{m}^{2}\right)=\mathrm{s} / \mathrm{m} \text {, }
$$

$\mathrm{M}$ is the NEO (comet) mass to be re-directed, and $\mathrm{F}$ is the radiant fluence at the comet surface. For the models used here the nuclear explosive generated radiation is primarily pulsed thermal radiation characterized by "Planckian" or "black body" radiation. Since only a portion of the radial expanding nuclear radiation will intercept the NEO surface, assuming a radially symmetric radiation pattern, the nuclear explosive must generate many times the required radiation energy, $\mathrm{E}$, to produce the desired momentum change in the NEO. 
To elaborate, the impulse (momentum) coupling coefficient $C_{M}$ for an energy delivery mechanism (interaction) is defined as the ratio of the target recoil impulse (impulse per unit area) to the energy (or energy per unit area) interacting on the target. Momentum for the entire system is conserved and in analogy to a rocket impulse propulsion hot gas and fine particle ejecta drive the target in the opposite direction.

Accurate estimates of $\mathrm{C}_{\mathrm{M}}$ require carefully controlled experiments such as those described in [1] and/or computer simulations of energy transfer integrals using a database of, for example, $\mathrm{X}$-ray absorption coefficients corresponding to nuclear radiation as has been done in [3]. Care must be taken to derive and apply impulse coupling values within a range of validity.

The amount of energy available for a comet deflection can be computed from the high energy density (HED) soft X-ray radiation source drivers from 0.25 to $4.0 \mathrm{keV}$. This radiation ablatively generates surface impulsive momentum coupling to model comet nuclei comprised of water ice $(70-100 \%$ mass fraction) and olivine $(0-30 \%)$. This first phase comet modeling is analogous to work initially achieved analytically by using a modified (MBBAY) BBAY type analysis [12 - 15] to compute momentum coupling from high fluence short pulse of scattered (mostly re-radiated) photon energy. Target (planar) materials are assumed cold and un-ionized with interaction properties unaffected by energy deposition (fluence). Time-independent transport and deposition of thermal X-ray spectra on materials and associated mechanical effects such as momentum coupling are determined. Photoelectric absorption is the primary target interaction but Compton scattering and fluorescence emission are also taken into account.

These MBBAY calculations support and guide the second (future) stage of this comet research that is to experimentally determine $C_{M}$ and the equation of state. Such an experimental determination requires measuring the net momentum imparted to the sample by a known X-ray pulse, but also provides pressure and average shock velocity measurements of the targets, which are not required for the impulse determination but are needed for the full hydrocode time dependent analysis of the interaction. Experimental confirmation of both the MBBAY and hydrocode analyses can be accomplished by using the Sandia National Laboratories (SNL) X ray emitting Z-pinch facility analogous to the research on meteorite targets $[1,15,16]$.

Spallation of comet target materials can potentially affect the momentum transfer. Spallation (solid scabs) on the irradiated side (target front surface) is unlikely because the heating is intense and no significant mechanism is present for causing tensile failure. In isolated low-fluence cases, some spall is possible; this would increase $\mathrm{C}_{\mathrm{M}}$, but this effect would be small and of little consequence. Spall scabbing at the back surface (far side of the target) is also possible, but not likely to be significant because the initial impulse is short, the transient pressure gradient originating at the target is steep [1] and hence the loading wave is strongly attenuated while transiting through the NEO. An exception would be the case of a pre-existing failure plane near the back free surface allowing the low-velocity separation of a large mass (no hot gas or fine particles). In any case, the momentum associated with the center of mass of the non-blowoff material will be unchanged. Therefore, in general the mass ejection in the irradiated zone is the dominant cause of recoil momentum and hence orbital change.

The nuclear explosive will provide a roughly isotropic energy distribution of thermal radiation, well characterized by Planckian or black-body continuum spectra. Although this 
irradiation will likely encounter irregular surface topography and possibly varied composition, the net thrust will not be strongly affected. Uncertainties could be quantified by Monte Carlo calculations [17].

\section{Momentum coupling analytic impulse model}

The X-ray momentum coupling coefficients, $\mathrm{C}_{\mathrm{M}}$, are determined from the ratio of the impulse coupling, Pa-s, divided by the fluence, $\mathrm{F}=\mathrm{J} / \mathrm{m}^{2}$ where $\mathrm{C}_{\mathrm{M}}=(\mathrm{Pa}-\mathrm{s}) / \mathrm{F}=\mathrm{s} / \mathrm{m}$. The model targets are assumed to be uniform, isotropic, and homogeneous with negligible angular momentum (torque). This analytic impulse model has been used to calculate blow-off impulse and subsequent momentum coupling effects from nuclear explosions [12, 13] on asteroids [3]. This research extends the analytic methodology to comet interactions. The impulse, I, is [12]

$$
I=\alpha \sqrt{ } 2\left[\int_{0}^{\mathrm{z} 0}\left\{E(z)-E_{0}\left(1+\ln \left(E(z) / E_{0}\right)\right)\right\} \rho^{2} z d z\right]^{1 / 2}
$$

where $1 \leq \alpha \leq \sqrt{ } 2$, generally taken as $\alpha=1.2$. $E(z)$ is the energy deposition per unit mass as a function of depth, $z$, in the target, and $E_{0}$ is the specific energy defining the extent of the material contributing to the blow-off impulse (i.e., where $E\left(\mathrm{z}_{0}\right)=E_{0}$ ), and $\rho$ is the target material density. More specifically, $E_{0}$ is the target melt and/or vaporization energy. In general, Eq. (2) will be numerically integrated over the target depth, $\mathrm{z}$ and the energy $\mathrm{E}(\mathrm{z})$. Integration is from the front surface, $z=0$, to the blow-off depth, $z=z_{0}$. If the energy dependent absorption coefficients,

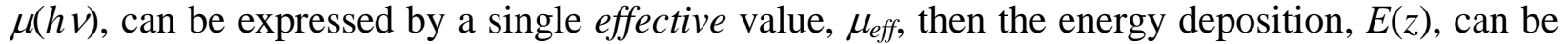
expressed as

$$
E(z)=\mu_{e f f} \Phi_{0} \exp \left(-\mu_{e f f} \rho z\right)
$$

where $\Phi_{0}$ is the incident energy fluence. In this case the MBBAY model is integrated to yield a relatively simple closed-form expression for the impulse. Representing the impulse in a nondimensional form, $I^{*}=\mu$ eff $I / E_{0}{ }^{1 / 2}$, as a function of the nondimensional fluence, $\Phi_{0}{ }^{*}=\mu_{e f f}$ $\Phi_{0} / E_{0}$, then equation (2) can be integrated in closed form, the result is [12]

$$
I^{*}=\alpha \sqrt{ } 2\left\{\Phi_{0}^{*}-\left[1+\ln \Phi_{0}^{*}+1 / 2\left(\ln \Phi_{0}\right)^{2}+1 / 6\left(\ln \Phi_{0}^{*}\right)^{3}\right]\right\}^{1 / 2}
$$

\section{High fluence limit}

In the limit of high fluence, $\Phi_{0} * \gg 1$ the logarithmic terms in the square brackets increase more slowly than the other terms, and equation (4a) reduces to

$$
\mathrm{I}^{*}=\alpha \sqrt{ }\left(2 \Phi_{0}^{*}\right), \Phi_{0}^{*}>1 .
$$

Changing back to dimensional variables this becomes

$$
\mathrm{I}=\alpha \sqrt{ }\left(2 \Phi_{0} / \mu_{\mathrm{eff}}\right), \Phi_{0}>E_{0} / \mu_{e f f},
$$


At high fluences the impulse is asymptotically independent of all material properties but the absorption coefficients, which are well-known, and scale simply with the square-root of the incident fluence.

\section{Application of the momentum coupling analytic impulse model}

There are three important properties of this model that apply for the approximate forms described above or for full numerical integrations over depth into the target and over spectral intensities and absorption coefficients. First, there is a deposited energy below which there is no material decomposition and thus no generated blowoff impulse. This assumes deposited energy is a monotonic decreasing function of the depth in the target as suggested above. For a given $\mathrm{x}-$ ray spectrum and target material this leads to a specific threshold for the incident energy fluence located at $\Phi_{0} *=1$. Second, if the impulse coupling coefficient, $C_{\mathrm{M}}$, is defined as the impulse, $I$, divided by the energy fluence incident on the surface of the target, $\Phi_{0}$, then $C_{M}$ will have a maximum value at a fluence roughly a factor of ten greater than the threshold, i.e., at $\Phi_{0} * \approx 10$. Finally, when the incident fluence is high-much greater than at the maximum coupling efficiency at the maximum of the impulse curve - the impulse will scale with the square-root of the incident fluence, as shown in equation 5 above. Uncertainty in target material decomposition energies will not be significant in this limit. For these high fluences the impulse will be relatively insensitive to the incident radiation, other than their X-ray absorption coefficients. For applications depending on maximum impulse coupling it is important to choose, if possible, an incident $x$-ray spectrum that maximizes coupling, which will be a Planckian spectrum of the form shown in Fig. 1.

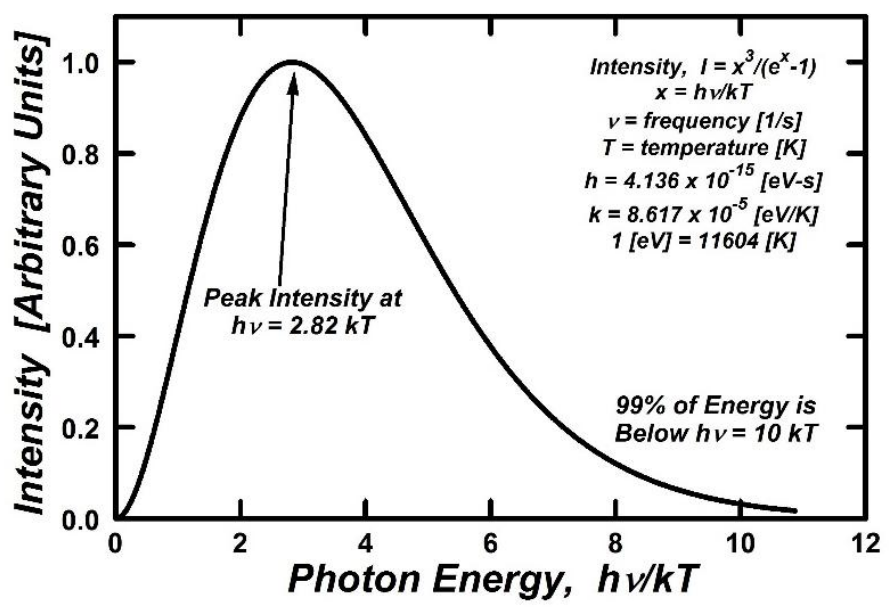

Figure 1. Thermal black body spectra. Pulsed thermal x-ray spectra can most easily be characterized by Planckian or black body representations as plotted here. For these continuous spectra the peak intensity occurs at photon energies about three times the thermal temperature kT, and virtually all the energy occurs below $10 \mathrm{kT}$. Although "effective" absorption coefficients can be used for limiting conditions, detailed numerical integration over this broad range of photon energies is generally required by the MBBAY impulse model. This is a consequence of the absorption coefficients for the target materials, which vary by factors of several thousand over this same range of photon energies. 
The same basic phenomenon that drives pulsed $\mathrm{x}$-ray momentum generation processes is the absorption of relatively low energy x-rays in the surface layers of the relevant targets. When the total broad and continuous x-ray fluence, extending over an order of magnitude in photon energies, is large enough target surface layers are explosively vaporized and generate an impulse to the target.

\section{Computational results}

The MBBAY integral computation results are summarized in Fig. 2(a) which shows scaled $\mathrm{X}$-ray energy deposition for a $100 \%$ ice comet and for a $70 \%$ ice and $30 \%$ olivine comet for Xray energies of $0.25,1.0$, and $4.0 \mathrm{keV}$ Planckian (black body) energy distribution. As expected the target penetration depth in the target is primarily dependent on the X-ray energy. Presence of the higher opacity olivine makes small changes to the energy deposition and thus has only a minor effect on the momentum coupling. Also, the heat of vaporization for olivine is almost three times that of ice $(9.0 \mathrm{~kJ} / \mathrm{kg}$, vs. $3.4 \mathrm{~kJ} / \mathrm{kg})$, leaving less absorbed energy for propelling ejecta and therefore for momentum generation.

Figure 2(b) shows the impulse on the $100 \%$ ice and $70 \%$ ice and $30 \%$ olivine comets as a function of fluence. At very high fluence the impulse is roughly linearly dependent on the fluence. Again, the effectiveness of penetration effects in the more transparent ice momentum transfer models suggests more energy is deposited at a greater depth. In the 100\% ice target the radiation penetrates deeper ablating more mass that contributes to greater comet ejecta. The higher energy X-ray radiation enhances impulse at greater fluence levels whereas the opposite is true at lower fluence.

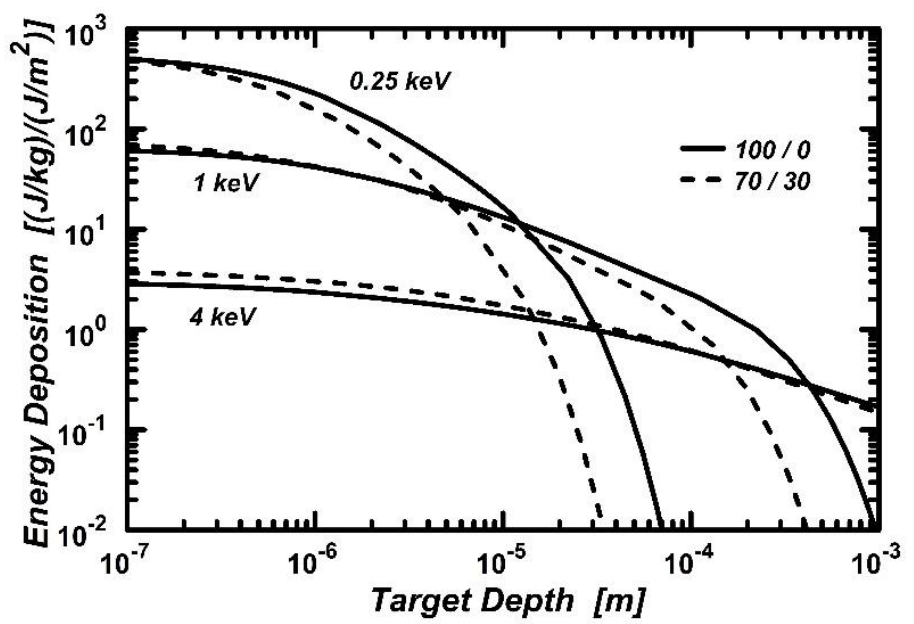

Figure 2(a). Energy deposition as a function of x-ray spectrum and target composition. The spectrum has a significant effect, but the composition of the comet target is of relatively small importance. Solid lines are for $100 \%$ ice and dashed curves are for $70 \%$ ice and $30 \%$ olivine. Note that because the deposition is dependent only on the target absorption coefficients, it can be scaled by the incident fluence. 


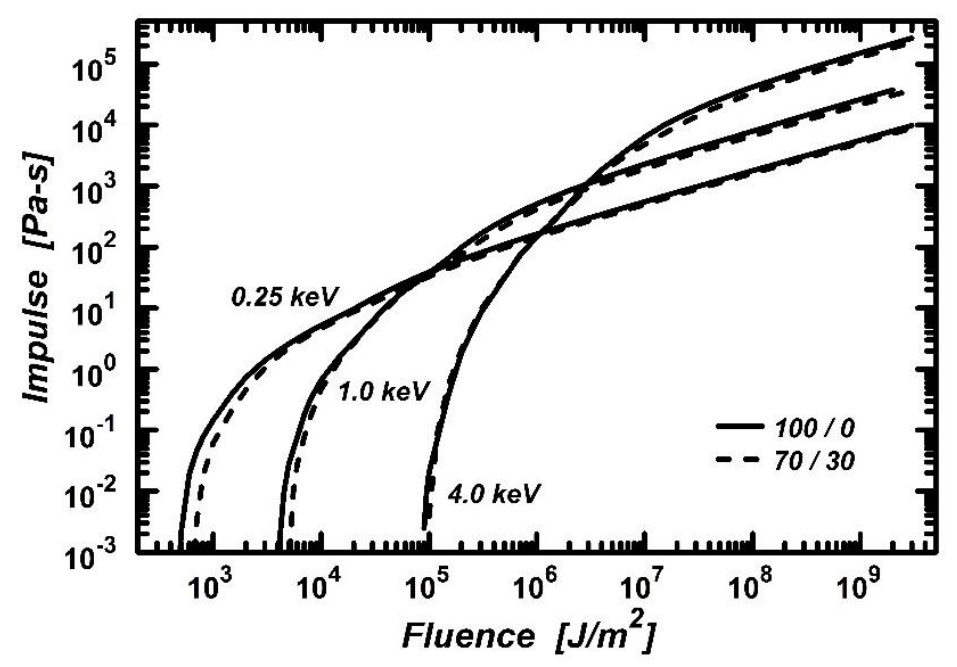

Figure 2(b). Impulse generation as a function of incident $x$-ray spectrum and target composition. Note both the threshold fluence for impulse generation as well as the simple square-root impulse scaling at high fluences. Solid curves (100/0) represent comet compositions of $100 \%$ ice, while dashed curves (70/30) indicate 70\% ice and 30\% olivine. As expected, the impulse or momentum generation shows a small dependence on the target composition.

Figure 3 shows the same impulse data only plotted in terms of the coupling coefficient. There are distinctive momentum coupling peaks for each of the spectra such that an increase in fluence does not necessarily increase the momentum coupling coefficients, $\mathrm{C}_{\mathrm{M}}$; it just less efficiently overdrives (heats) the target. We note that the maximum efficiency of the coupling is at relatively low fluences only a decade or two from the threshold fluence. In an operational interception scenario at high $\mathrm{F}, \mathrm{C}_{\mathrm{M}}$ and $\mathrm{F}$ must be optimized. An advantage of interception at high $\mathrm{F}$ where the impulse scaling goes as the square root there is less uncertainty as compared to applying the exact $\mathrm{F}$ to achieve $\left(\mathrm{C}_{\mathrm{M}}\right)_{\max }$ which is peaked.

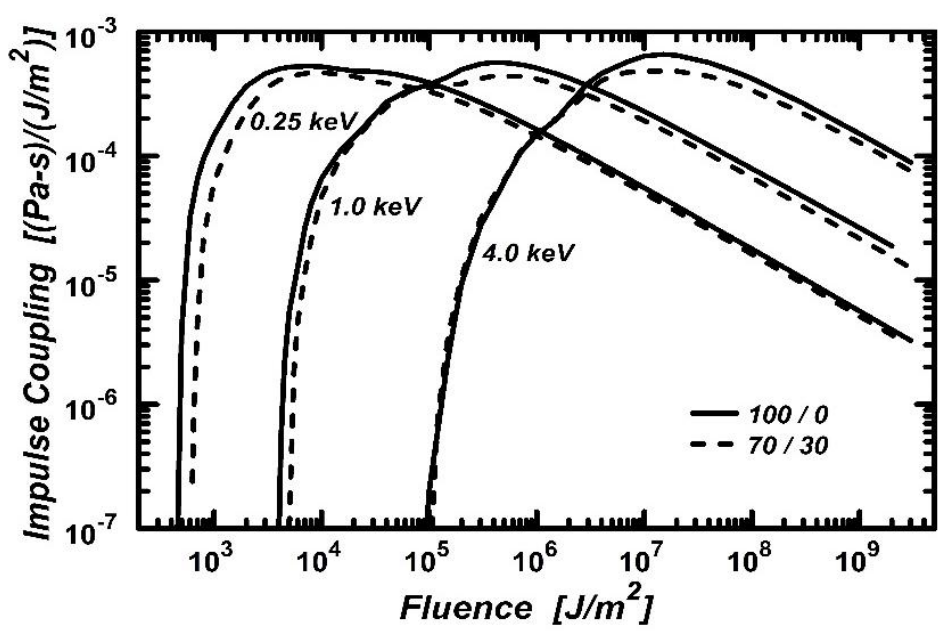

Figure 3. Impulse coupling as a function of incident spectra and target composition. There are three important aspects of this behavior; first is the threshold for impulse generation; second is the peak coupling or efficiency; and third is the square-root scaling of the impulse coupling at high 
fluences. Again the solid curves are for $100 \%$ ice and the dashed curves are for $70 \%$ ice and $30 \%$ olivine. As with the impulse, the curves are similar, only shifted to the right, roughly in proportion to the square of the temperature $\mathrm{kT}$.

Once again, the presence of olivine has a minor diminishing effect on the momentum coupling because more energy is absorbed at the surface by olivine with a greater opacity than ice. The peak $\mathrm{C}_{\mathrm{M} \text { max }}$ value of the comet model is about $50 \times 10^{-5}(\mathrm{~Pa}-\mathrm{s}) / \mathrm{F}(\mathrm{s} / \mathrm{m})$ for all of the source spectra radiation. This is about an order of magnitude greater for the ideal comet models than for the meteorite and planetary materials computer modeled and also experimentally subjected to the same X-radiation fluence [1]. This much higher coupling with ice is due to its lower vaporization energy and greater transparency. Also, the MBBAY computer models for meteorite, planetary, and comet targets assume uniform, homogeneous and isotropic targets similar to the present analysis, so the computed $\mathrm{C}_{\mathrm{M}}$ are expected to run higher than the experimental values.

There are asymptotic values of the impulse for the pure ice target and the $30 \%$ olivine for three $\mathrm{x}$-ray energies in the high fluence asymptotic region where the different values of impulse to the target converge.

\section{Conclusions}

The primary conclusion that may be drawn from these MBBAY calculations on the two putative comet models is that water ice has an X-ray momentum coupling coefficient an order of magnitude higher than do meteoritic and planetary materials. Bodies comprised of $100 \% \mathrm{H}_{2} \mathrm{O}$ or $70 \% \mathrm{H}_{2} \mathrm{O}$ and $30 \%$ distributed olivine powder subjected to high fluence soft $\mathrm{X}$-ray radiation $\left(\sim 10^{4}-10^{8} \mathrm{~J} / \mathrm{m}^{2}\right.$ at 0.25 to $\left.4.0 \mathrm{keV}\right)$ have maximum momentum couplings $\left(\mathrm{C}_{\mathrm{M}}\right)_{\max }$ at $50 \times 10^{-5}$ $\mathrm{s} / \mathrm{m}$; this compares with computed MBBAY values for meteoritic and planetary materials whose calculated and experimental values are respectively $\mathrm{C}_{\mathrm{M}}=2.7-5.4$ and $0.3-3.0 \times 10^{-5} \mathrm{~s} / \mathrm{m}$ [1]. The coupling is slightly lower for the ice comet with the $30 \%$ olivine than for the $100 \%$ ice comet. This result is not surprising given the higher olivine vaporization energy over ice by almost a factor of three, leaving less energy for the ejecta. Also, a higher opacity of the olivine over the water ice allows less penetration of the radiation in olivine.

Overall, for both meteorite and planetary materials models the MBBAY calculation give about $10 \%$ higher $\mathrm{C}_{\mathrm{M}}$ values than the experiments, although there is scatter in this data. Until proven by experiment it is assumed the MBBAY computations on the ideal comet models are also $10 \%$ higher than the experimental values for the reasons stated above, giving a robust $\left(\mathrm{C}_{\mathrm{M}}\right)$ $\max \sim 50 \times 10^{-5} \mathrm{~s} / \mathrm{m}$. However, operational fluences are likely to be higher than those at maximum efficiencies. Using this result, for example, if a comet of diameter $2 \mathrm{~km}$, mass $\mathrm{M} \sim 2.5 \times 10^{12} \mathrm{~kg}$ and density $600 \mathrm{~kg} / \mathrm{m}^{3}$ on a collision course with Earth that requires a velocity change of $1 \mathrm{~cm} / \mathrm{s}$ to avoid impact; using (1) it would require $5 \times 10^{13} \mathrm{~J}$ of energy or the equivalent of $\sim 12 \mathrm{kT}$ (kilotons) of TNT yield normal to the comet surface. This is roughly the total yield of the first atomic bomb dropped on Hiroshima. Accordingly, a $20 \mathrm{~km}$ comet requiring a velocity change of $2 \mathrm{~cm} / \mathrm{s}$ to avoid Earth impact would require $10^{17} \mathrm{~J}$ of energy or the equivalent of $\sim 20 \mathrm{MT}$ (megatons) of TNT normal to the comet surface. 
Depending on the stand-off distance geometry, the radial irradiation fields of a nuclear explosive blow-off yields would have to be roughly an order of magnitude greater than the fluence on the comet targets surface requiring total yields of $\sim 120 \mathrm{kT}$ and 200 MT TNT equivalent, respectfully. Although this energy may be required to be delivered by a combination of optimal stand-off distance, directed energy, and in repetitive stand-off blasts, these numbers are well within the reach of current nuclear technology.

There is another caveat when designing mitigation missions to NEO comets which is based on the comet's level of emission activity. Higher surface emission activity can complicate efforts to obtain a size (mass) albedo relationship because then there is not necessarily a strong or reliable correlation between reflected light and mass. Nonetheless, based on these calculations we conclude that given enough time, utilization of robust astronautically conventional or nuclear rocket systems, and adequate nuclear payloads with repetitive standoff capabilities nuclear X-ray radiation is a viable option for deflecting a comet with an orbit trajectory potentially hazardous to Earth. An added advantage of this approach is that the technology to carry out a nuclear deflection is available.

\section{Acknowledgments}

Sandia National Laboratories is a multi-mission laboratory managed and operated by Sandia Corporation, a wholly owned subsidiary of Lockheed Martin Corporation, for the U.S. Department of Energy's National Nuclear Security Administration under contract DE-AC0494AL85000.

\section{References}

1. Remo, J. L., Furnish, M. D., and Lawrence, R. J., "Plasma driven Z-pinch X-ray loading and momentum coupling in meteorite and planetary materials," Plasma Phys., pp 1-21, 2013.

2. Schafer, B. P., Garcia, M. D., Managan, R. A., Remo J. L., Rosenkilde, C. E., Scammon, R. J., Snell C. M., and Stellingwerf, R. F. "Momentum coupling to NEOs. In Near Earth Objects: The United Nations International Conference (Annals of the New York Academy of Science, vol 822 (ed. J. L. Remo). New York: New York Academy of Science, pp. 552-565, 1997.

3. Hammerling P. and Remo J. L., "NEO Interaction with Nuclear Radiation," Acta Astr. 36, pp 337-346, 1995.

4. Remo, J. L., "Classifying and modeling NEO material properties and interactions," in Hazards Due to Comets and Asteroids, Univ. Arizona Press, T. Gehrels ed. 551-596, 199.

5. Bombardelli, C., Amato, D. and Cano, J. L., "Mission analysis for the ion beam deflection of fictitious asteroid 2015PDC," Acta Astronautica 118 (2016), 296-307. 
6. Deng, H., Li, T., and Wang, Z., "Pretension design for space deployable mesh reflectors under multi-uncertainty," Acta Astronautica, 115 (2015), 270-276.

7. Schmit, M., Fasoulas, S., and Utzmann, J., "Performance model for space-based laser debris sweepers,"Acta Astronautica, 115 (2015), 376-383.

8. Cheng, A. F., et al, "Asteroid impact \& deflection assessment mission: Kinetic impactor," Planetary and Space Science, 121 (2016), 27-35.

9. Taylor, M. G. G. T. et al, “Rosetta begins its comet tail,” Science 347, 387, 2015.

10. Mumma, M. J. and Charnley, S. B., "The chemical composition of comets-emerging taxonomies and natal heritage," Ann. Rev. Astron. Astrophys., 49, 475-534, 2011.

11. Altwegg, K. et al, "67P/Churyumov-Gerasimenko, a Jupiter family comet with a high $\mathrm{D} / \mathrm{H}$ ratio,” Science 347, 1261952, 2015.

12. Lawrence, R. J., "The equivalence of simple models for radiation-induced impulse," pp. 785788 in Shock Compression of Condensed Matter, 1991, S.C. Schmidt, R. J. Dick, J. W. Forbes, and D. G. Tasker, eds., North-Holland Publishers, 1992.

13. Lawrence, R. J., "Analysis of the Interaction of Short Pulse High Fluence Radiation with Targets," pp. 1185-1188 in Shock Compression of Condensed Matter, 1999, M. D. Furnish et al eds., American Institute of Physics, Woodbury N. Y., 2000.

14. Lowen, R., Schaibly, J. and Stephens, T. "X-ray radiation transport program (users) handbook"' Defense Nuclear Agency, Alexandria, VA, 1993.

15. Remo, J. L. and Furnish, M. D., "High intensity x-ray coupling to meteorite targets." In: Shock Compression of Condensed Matter-2001 (eds. M. D. Furnish, N. N. Thadhani, and Y. Horie)), AIP Press, pp 1163-1168, 2002.

16. Remo, J. L. and Furnish, M. D. "Analysis of Z-Pinch shock wave experiments on meteorites and planetary materials.” Int. J. Impact Eng 35, pp 1516-1521, 2008.

17. Bazzocchi, M. C. F. B. and M. Reza Emami, "Comparative analysis of redirection methods for asteroid resource exploitation, Acta Astr, 20, 1-19, 2016. 